\title{
Very forward energy distributions and jet production observed with CASTOR in CMS
}

\author{
Alex Van Spilbeeck* \\ Universiteit Antwerpen \\ E-mail: Alex.VanSpilbeeck@UAntwerpen.be
}

The CASTOR calorimeter at the CMS experiment at LHC provides very forward $(-6.6<\eta<$ $-5.2)$ energy measurements. Jets in this regime can be used as very sensitive probes of the low- $x$ parton dynamics in the proton. Measurements of deposited energy in this region can be used to model cosmic ray induced extensive air showers at ultra-high energies. The spectra of jets in CASTOR and the total, electromagnetic and hadronic energy depositions in CASTOR are measured and corrected to particle level, and compared to various relevant Monte Carlo models.

XXIV International Workshop on Deep-Inelastic Scattering and Related Subjects

11-15 April, 2016

DESY Hamburg, Germany

${ }^{*}$ Speaker. 


\section{Introduction}

The majority of processes taking place in high energy proton-proton interactions are QCDmediated parton-parton scatterings. These processes create a huge background for all processes and searches studies at the LHC and must be modelled precisely to separate such events from possible new physics events. QCD processes can be described to great precision with DGLAP part evolution equations and collinear factorization [1, 2, 3, 4]. Due to approximations made it is expected that the predictions of this method will break down at very low $x$ values, but no precise conditions for this breakdown can be given. Events with low $x$ are characterized by very forward (high $\eta$ ), low- $p_{T}$ jets.

The performance of Monte Carlo models in the very forward regime is a very important benchmark for studies in the field of astroparticle physics due to the very forward boost of the outgoing energy. This environment is very similar to collisions of ultra-high energy cosmic particles with stationary atmospheric particles (atomic nuclei) which result in extensive air showers [5]. These showers contain muons through the decay of mesons coming from the initial collision, but the production mechanism of these muons is a major mystery in the field [6].

\section{Experimental setup}

The central feature of the Compact Muon Solenoid (CMS) apparatus is a superconducting solenoid of $6 \mathrm{~m}$ internal diameter, providing a magnetic field of 3.8 T. Within the field volume are a silicon pixel and strip tracker, a lead tungstate crystal electromagnetic calorimeter (ECAL), and a brass/scintillator hadron calorimeter (HCAL). Muons are measured in gas-ionization detectors embedded in the steel return yoke. Extensive forward calorimetry complements the coverage provided by the barrel and endcap detectors. The central detectors of CMS are complemented by calorimeters in the forward direction, which are all relying on the production of Cherenkov photons by charged particles in quartz. The "hadron forward" (HF) calorimeters cover the pseudorapidity interval $3<|\eta|<5.2$ and are using quartz fibers embedded in a steel absorber. A more extensive description can be found in [7]. In the very-forward direction there is the CASTOR calorimeter located at a distance of $14.2 \mathrm{~m}$ from the interaction point at a radial distance from the LHC beam of about 4 to $15 \mathrm{~cm}$. This corresponds to a pseudorapidty coverage of $6.6<\eta<5.2$. CASTOR is a sampling calorimeter using layers of fused silica quartz plates and tungsten absorbers. CASTOR is segmented in 14 longitudinal and 16 azimuthal channels. The first two front channels correspond to a combined depth of $20 X_{0}$ and are used as the electromagnetic section, while the full depth of the calorimeter amounts to $10 \lambda_{I}$. The data of CASTOR are reconstructed in 16 towers, each summing up the 14 longitudinal channels at the same azimuthal location. Towers are zero suppressed with $650 \mathrm{MeV} \sqrt{N_{\text {channel }}}$ by considering the noise level in each of the $N_{\text {channel }}$ channels used to construct the tower. As a last step, towers are clustered into jets with the anti- $k_{T}$ algorithm [8] using a radius parameter $R=0.5$. Because of the very forward position of CASTOR, it does not have an $\eta$-segmentation: all jets are assigned a default $\eta=-5.9$ value, which corresponds to the value center of the calorimeter.

CASTOR can distinguish between deposits of electromagnetic energy and deposits of hadronic energy by measuring it in different modules. 
The response of the detector is simulated using the GEANT4 framework [9] and is used to correct from measured spectra to particle level spectra. The Monte Carlo models used during the analyses are PYTHIA8 (version 8.212) [10] with tune CUETP8M1 [11] and MBR [12] model as well as EPOS-LHC [13].

\section{Data analysis}

For the measurement of the spectra $0.212 / \mathrm{nb}$ of collected data were used. These were collected at the beginning of the second LHC run when the CMS magnet was turned off.

\subsection{Energy measurement}

Data was selected by monitoring activity in the HF calorimeters. The influence of electronic noise in CASTOR is minimized by requiring at least one tower in either of the HF calorimeters (on negative or positive side of the interaction point) above $5 \mathrm{GeV}$ : this reduces sensitivity to noise to less than $1 \%$. The total energy measured by CASTOR is taken as the sum of all calorimeter towers above the noise threshold (Sec. 2). The sum of energy of towers in the two frontmost modules is the electromagnetic fraction of energy deposited in CASTOR, towers in the twelve backmost sum up to form the hadronic fraction of the energy.

The energy spectra are corrected to stable particle level, where stable means a lifetime $c \tau>$ $1 \mathrm{~cm}$. Stable particles are assigned to system $X$ or system $Y$, with these systems lying on opposite sides of the largest rapidity gap in the event. A variable $\xi_{S D}$ is then defined as:

$$
\begin{gathered}
\xi_{X}=M_{X}^{2} / s \\
\xi_{Y}=M_{Y}^{2} / s \\
\xi_{S D}=\max \left(\xi_{X}, \xi_{Y}\right) .
\end{gathered}
$$

Events with $\xi_{S D}>10^{-6}$ are selected.

Data energy spectra are corrected to stable particle level with calibration to counter the noncompensation of CASTOR and are unfolded with the d'Agostini iterative procedure with early stopping [14].

\subsection{Jet energy spectrum}

To correct for the non-compensating nature of the CASTOR calorimeter, jet energies were calibrated. The calibration factors were determined from Monte Carlo simulations that incorporate the calorimeter response: essentially the jet energies are scaled up with approximately $40 \%$.

In a following step the measured and calibrated jet energy spectrum was unfolded. To this end, a response matrix was constructed in Monte Carlo with reconstructed CASTOR jets and generator level jets (anti- $k_{T}$ with radius parameter $R=0.5$ ). Jets are matched by looking at the most energetic detector level jet and matching this to the most energetic generator level jet with a maximum difference in azimuthal angle $\Delta \phi_{\max }$, and iterating these steps until no more matches can be made. Each jet cannot be matched more than once. Non-matched generator level and detector level jets ("misses" and "fakes") impose a model dependence on the result of the unfolding procedure: their influence can be reduced by setting $\Delta \phi_{\max }=0.5$ and considering only generator level jets in $-6.6<$ 
$\eta<-5.2$ in the matching procedure. At $p_{T}>3 \mathrm{GeV}$ the fractions of misses and fakes are both below $10 \%$, and for $p_{T}>5 \mathrm{GeV}$ these values are less than $1 \%$.

The measured spectrum was unfolded with the d'Agostini iterative procedure with early stopping [14]. It was found that 80 iterations the unfolding bias is small enough, and Poisson-induced statistical fluctuations do not yet contribute to the result.

\section{Uncertainties}

Several sources of systematic uncertainties were found and taken into account.

- CASTOR energy scale. CASTOR is cross-calibrated via a measurement of energy in $3<$ $|\eta|<5$ which is extrapolated with a large set of Monte Carlo models to $5.2<|\eta|<6.6$. This is the largest uncertainty in both measurements.

- Luminosity.

- Model dependence. The model dependence is evaluated by unfolding the data with three different models (PYTHIA8 (CUETPM1), PYTHIA8 (MBR) and EPOS). The depence ranges between $20 \%$ and $50 \%$.

- Position uncertainty. The true position of CASTOR is known with a precision of $1 \mathrm{~mm}$, and it can vary up to $1 \mathrm{~cm}$ over the period during whihc data is taken. Changes in position change the true $\eta$ acceptance of the calorimeter, and have an influence of $10 \%$ to $30 \%$.

\subsection{Results}

\subsection{Energy measurement}

Figures 1, 2 and 3 show the respectively the total, electromagnetic and hadronic energy spectra after calibration and unfolding. The spectra are compared to cosmic ray Monte Carlo models (left panel) and PYTHIA and HERWIG (right panel).

\subsection{Jet energy spectrum}

Figures 4 and 5 show the final unfolded spectrum of the data compared to several Monte Carlo model predictions. It is clear that the uncertainty on the CASTOR energy scale has a large influence on the final uncertainty of the differential cross section but is significantly smaller when considering the yield per event. At low $p_{T}$ values the influence of MPI on the cross section is clearly visible.

\section{Conclusions}

In both measurements the CASTOR energy scale is large and increases at higher energies.

None of the models describes the shape of all observations. QGSJETII.4 and SIBYLL 2.3 describe data best overall while PYTHIA 8 4C+MBR and HERWIG++ UE-EE-5C perform worst. The electromagnetic energy spectrum is described best by all models. The influence of multi-parton interactions and the underlying event are clear.

The jet energy spectra correspond with the the data due to the large uncertainty. PYTHIA tends to predict a harder spectrum, QGSJETII.4 and EPOS indicate a slightly sofer spectrum. Overall EPOS yields the best agreement. 

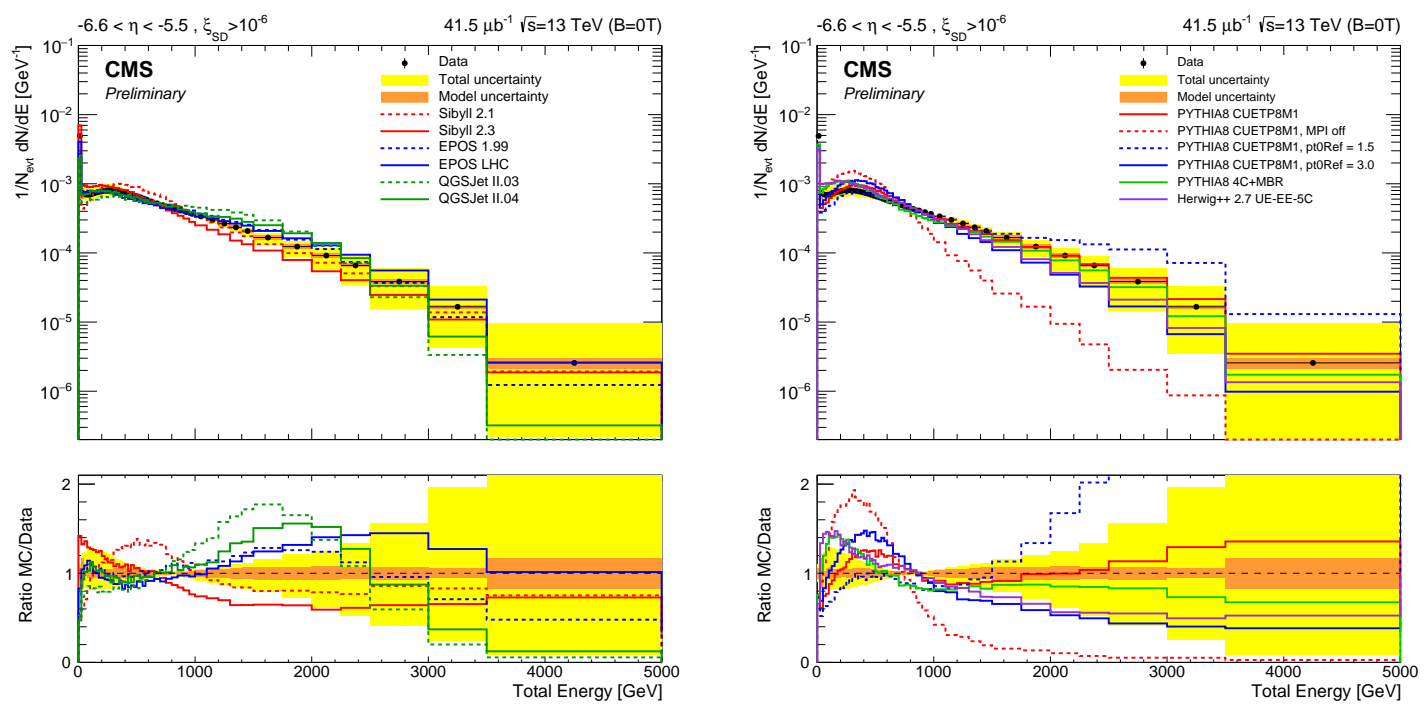

Figure 1: Normalized total energy spectrum in the acceptance of CASTOR for events with $\xi_{S D}>10^{-6}$.
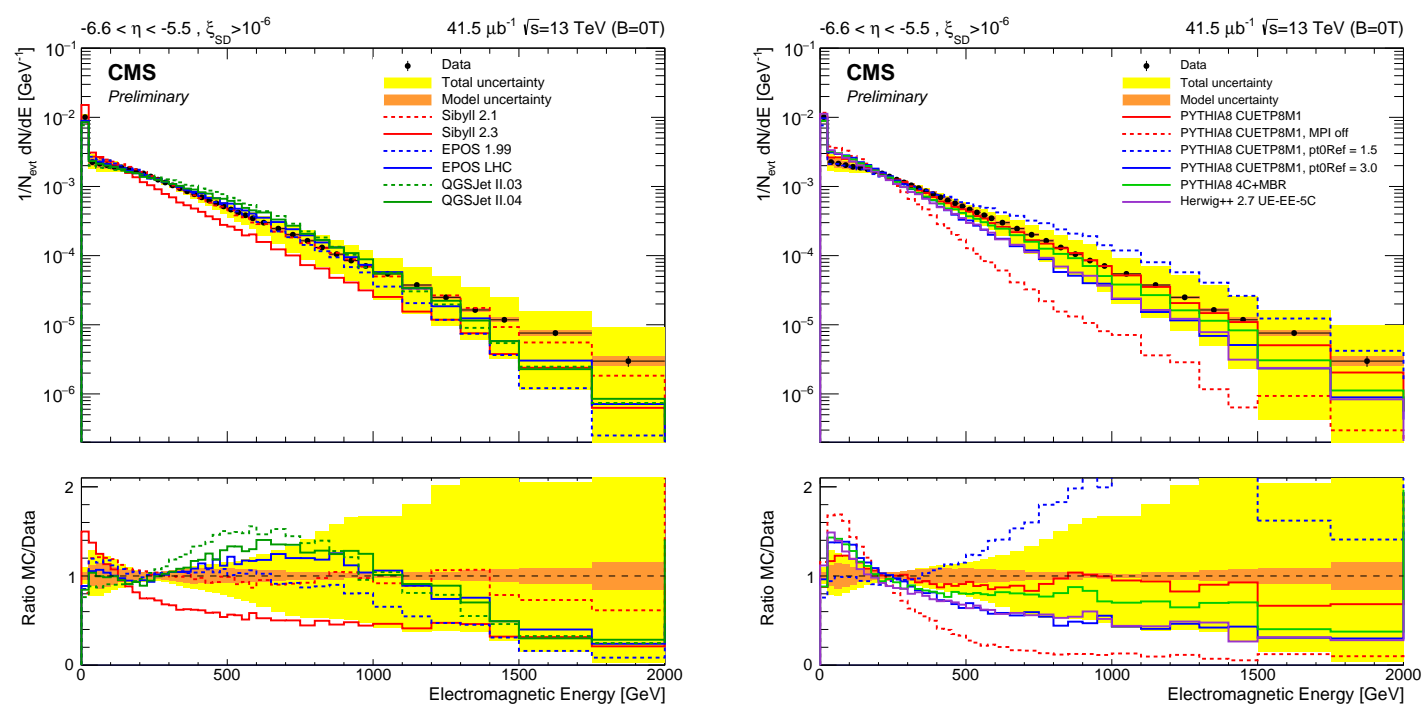

Figure 2: Normalized electromagnetic energy spectrum in the acceptance of CASTOR for events with $\xi_{S D}>10^{-6}$.

\section{References}

[1] V. N. Gribov and L. N. Lipatov, Deep inelastic ep scattering in perturbation theory, Sov. J. Nucl. Phys. 15 (1972) 438.

[2] L. N. Lipatov, The parton model and perturbation theory, Sov. J. Nucl. Phys. 20 (1975) 94.

[3] G. Altarelli and G. Parisi, Asymptotic freedom in parton language, Nucl. Phys. B126 (1977) 298.

[4] Y. L. Dokshitzer, Calculation of the structure functions for deep inelastic scattering and $e^{+} e^{-}$ annihilation by perturbation theory in quantum chromodynamics, Sov. Phys. JETP 46 (1977) 641. 

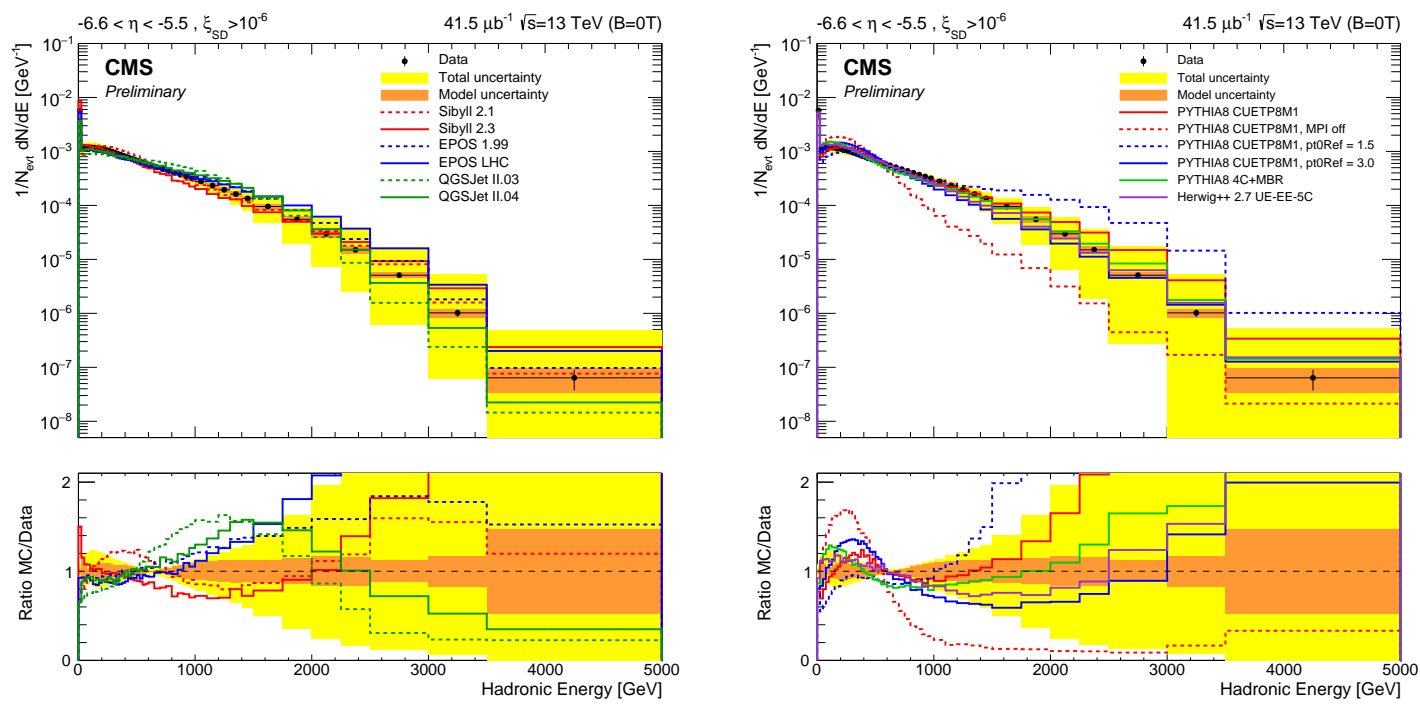

Figure 3: Normalized hadronic energy spectrum in the acceptance of CASTOR for events with $\xi_{S D}>10^{-6}$.
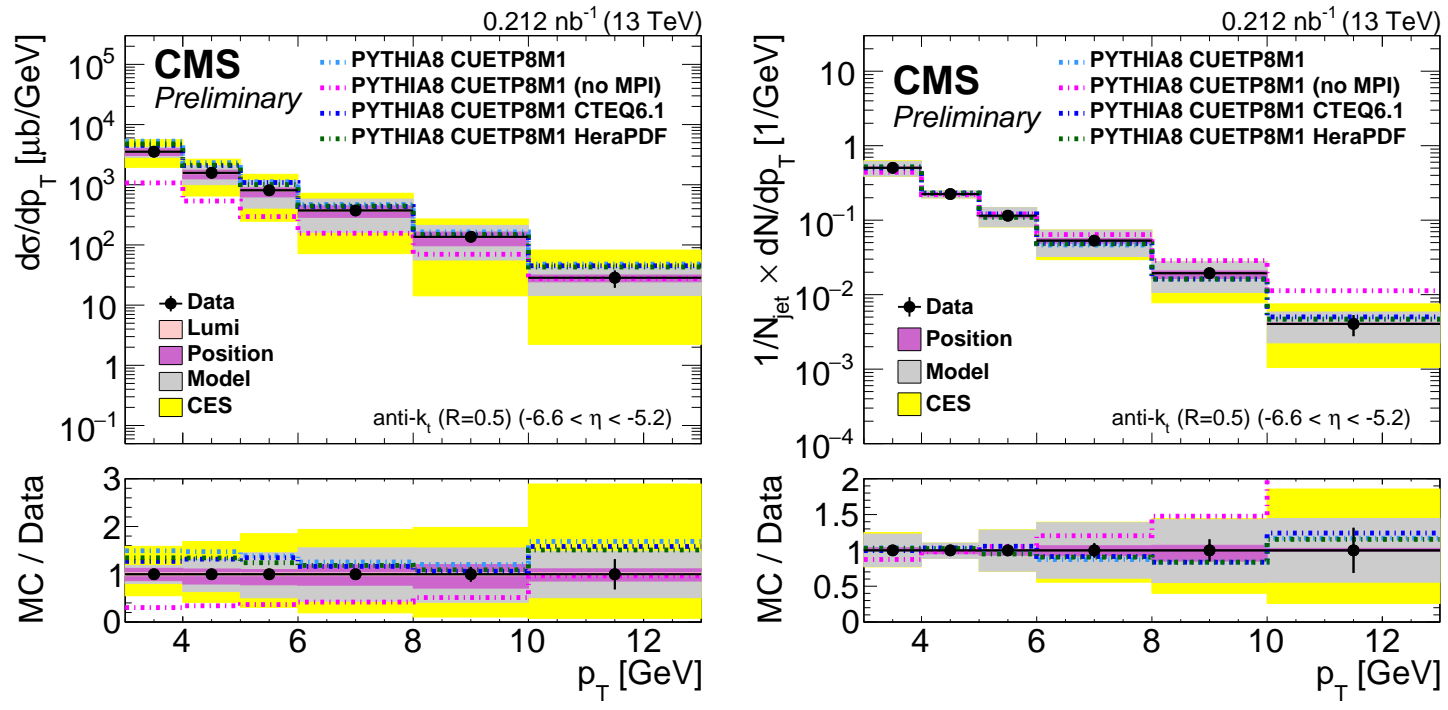

Figure 4: Final unfolded differential jet- $p_{T}$ spectrum in CASTOR compared to different model predictions. Left panel: the cross section. Right panel: the jet yield per event.

[5] R. Ulrich, R. Engel, and M. Unger, Hadronic Multiparticle Production at Ultra-High Energies and Extensive Air Showers, Phys. Rev. D83 (2011) 054026,

[doi:10.1103/PhysRevD.83.054026,arXiv:1010.4310].

[6] Pierre Auger Collaboration, Muons in air showers at the Pierre Auger Observatory: Mean number in highly inclined events, Phys. Rev. D91 (2015), no. 3, 032003,

[doi:10/1103/PhysRevD.91.05991,10.1103/PhysRevD.91.032003,arXiv:1408.1421]. [Erratum: Phys. Rev. D91, no.5, 059901 (2015)]

[7] CMS Collaboration, The CMS experiment at the CERN LHC, JINST 3 (2008) S08004, [doi:10.1088/1748-0221/3/08/s08004]. 

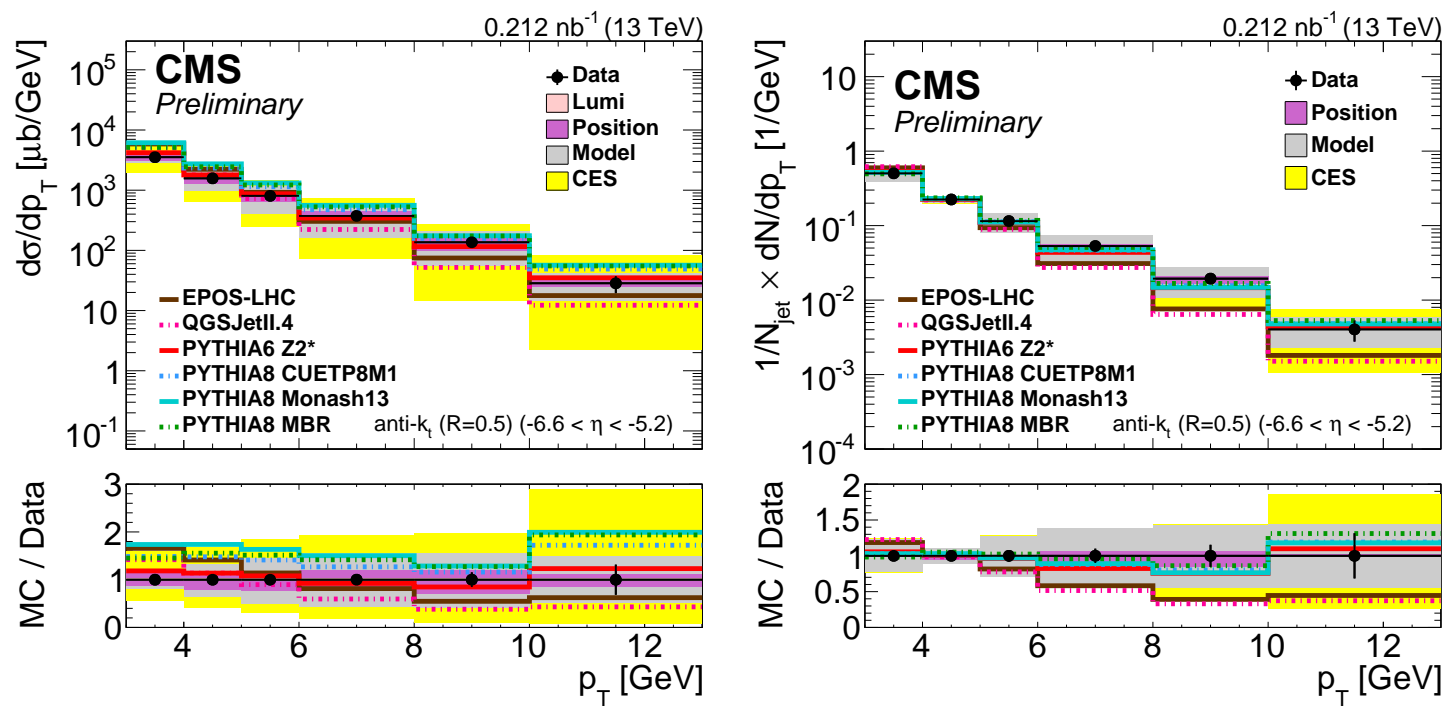

Figure 5: Final unfolded differential jet- $p_{T}$ spectrum in CASTOR compared to different model predictions. Left panel: the cross section. Right panel: the jet yield per event.

[8] M. Cacciari, G. P. Salam, and G. Soyez, The anti-kt jet clustering algorithm, JHEP 04 (2008) 063, [doi:10.1088/1126-6708/2008/04/063,arXiv:0802.1189].

[9] S. Agostinelli et al., Geant4a simulation toolkitâĂİ, Nuclear Instruments and Methods in Physics Research Section A: Accelerators, Spectrometers, Detectors and Associated Equipment 506 (2003), no. 3, 250 - 303, [doi: http: / /dx.doi.org/10.1016/s0168-9002 (03) 01368-8].

[10] T. Sjstrand et al., An Introduction to PYTHIA 8.2, Comput. Phys. Commun. 191 (2015) 159-177, [doi:10.1016/j.cpc.2015.01.024,arXiv:1410.3012].

[11] CMS Collaboration, Event generator tunes obtained from underlying event and multiparton scattering measurements, Eur. Phys. J. C76 (2016), no. 3, 155,

[doi:10.1140/epjc/s10052-016-3988-x, arXiv:1512.00815].

[12] R. Ciesielski and K. Goulianos, MBR Monte Carlo Simulation in PYTHIA8, PoS ICHEP 2012 (2013) 301, [arXiv:1205.1446].

[13] T. Pierog et al., EPOS LHC: Test of collective hadronization with data measured at the CERN Large Hadron Collider, Phys. Rev. C92 (2015), no. 3, 034906,

[doi:10.1103/PhysRevC.92.034906,arXiv:1306.0121].

[14] G. D’Agostini, A Multidimensional unfolding method based on Bayes' theorem, Nucl. Instrum. Meth. A362 (1995) 487-498, [doi : $10.1016 / 0168-9002$ (95) 00274-X]. 